\title{
Quantifying the Impact of Leveraging and Diversification on Systemic Risk
}

\author{
Paolo Tasca ${ }^{\star}$, Pavlin Mavrodiev, Frank Schweitzer \\ Chair of Systems Design, ETH Zurich, Weinbergstrasse 58, 8092 Zurich, Switzerland
}

\begin{abstract}
Excessive leverage, i.e. the abuse of debt financing, is considered one of the primary factors in the default of financial institutions. Systemic risk results from correlations between individual default probabilities that cannot be considered independent. Based on the structural framework by Merton (1974), we discuss a model in which these correlations arise from overlaps in banks' portfolios. Portfolio diversification is used as a strategy to mitigate losses from investments in risky projects. We calculate an optimal level of diversification that has to be reached for a given level of excessive leverage to still mitigate an increase in systemic risk. In our model, this optimal diversification further depends on the market size and the market conditions (e.g. volatility). It allows to distinguish between a safe regime, in which excessive leverage does not result in an increase of systemic risk, and a risky regime, in which excessive leverage cannot be mitigated leading to an increased systemic risk. Our results are of relevance for financial regulators.
\end{abstract}

Keywords: Systemic risk, Leverage, Diversification

JEL classification: G20, G28

\section{Introduction}

Systemic risk can be generally described as the risk that a failing agent (e.g. a bank in a financial system or a firm in a supply system) causes the failure of other agents such that failing cascades may encompass the whole system (Battiston et al., 2009b). This approach differs from other notions of risk (Embrechts et al., 2011) which treat the default of individual agents as an extreme event for which the probability is calculated regardless of the interaction among economic agents. Instead, a systemic notion of risk requires to explicitly take into account two ingredients: (a) the stability condition of individual agents, and (b) the impact of interactions between agents on their mutual stability. In this paper, we apply this systemic perspective to analyze the stability of a banking system, dependent on the leverage and diversification of individual banks.

\footnotetext{
${ }^{0}$ The authors acknowledge financial support from the ETH Competence Center "Coping with Crises in Complex Socio-Economic Systems" (CHIRP 1 grant no. CH1-01-08-2), the European FET Open Project "FOC" (grant no. 255987), and the Swiss National Science Foundation project "OTC Derivatives and Systemic Risk in Financial Networks" (grant no. CR12I1-127000/1). ( $\star$ ) Correspondence to Paolo Tasca, ptasca@ethz.ch.
} 
The stability of a single bank is commonly expressed by the composition of its balance sheet and measured by the leverage, e.g. by the debt-to-asset ratio. To quantify stability, we calculate the individual default probability using the well established structural framework by Merton (1974) in which the firm's leverage plays a major role. This approach has become popular among both academicians and practitioners thanks to its tractability and simplicity. For a survey see (Bohn, 2000). Moreover, the Financial Stability Board (2010) recommends it as a building block in establishing a regulatory framework that can cope with risk from systemic linkages.

These linkages arise from bilateral exposures (e.g. mutual claims) and common assets which may lead to interlocking balance sheets (see Kiyotaki and Moore, 2002). Including these effects into a systemic notion of risk requires explicit knowledge of banks' balance sheets and their change over time. This information is mostly confidential, due to strategic issues, and therefore unattainable for a system comprised of many banks. To avoid the trap of insufficient data, we study the mutual impact of failing banks by calculating their joint default probability (hereafter, "systemic default probability") based on their individual default probabilities, as defined by the Merton framework, and their asset correlation. We assume that risk-averse banks hold diversified portfolios in order to mitigate their individual default risk. Therefore, in a market with a finite number of (uncorrelated) investment opportunities, also called projects, asset correlation among banks increases with diversification due to overlapping portfolios. Stein (2009) identifies similar portfolios, or "crowding", as a destabilizing mechanism in financial markets.

In our paper, we particularly focus on the impact of excessive leverage and diversification on the systemic default probability and their possible interdependence. Excessive leverage refers to the practice of banks to engage in huge debt to buy more assets, in order to increase their return on equity. As the Financial Service Authority (FSA, 2009) and the Financial Stability Board (FSB, 2009) jointly point out, excessive leverage by banks increases systemic risk. However, our hypothesis is that diversification can compensate some of the hazards of excessive leverage, under optimal conditions. In our paper we identify two different regimes: (i) a safe regime in which an increase of leverage can be compensated by a proper diversification strategy, leading to a low systemic default probability, and (ii) a risky regime in which the same increase of leverage will lead to an increase of the systemic default probability, because the diversification strategy is not adequate and/or the market conditions are adverse. The latter consider, in our model, the size of the market, the market risk as measured by the volatility, and the time horizon.

The boundary between the two regimes is, in our model, given by a critical level of diversification, $n^{\star}\left(f_{n}, f_{a}, N, \chi\right)$, which depends on the market size $N$, the market conditions, $\chi$ (which also include the volatility and the time horizon), and two leverages, referring to a normal $\left(f_{n}\right)$ and an abnormal $\left(f_{a}\right)$ level. The safe regime is given by $n \geq n^{\star}$, whereas the risky regime is the opposite. We show that $n^{\star}$ increases with $N$. This seems to be counter-intuitive because the larger the market, the less should be the portfolio overlap for a given level of diversification. However, this 
only holds if $n$ is above the critical level of diversification. With increasing market size, banks have to reach a larger level of diversification in order to be on the safe side. Secondly, we show that excessive leverage expands the risky regime and therefore the critical level of diversification. Interestingly, there is not always a combination of leverage and diversification to reach the safe regime. Even with increasing market size, the market conditions may prevent this, thus the systemic default probability is not reduced.

Our paper contributes to several research lines. First, we show a different way of how the well established Merton (1974) model can be extended to joint defaults. Second, we extend the previous literature on the optimal diversification level pioneered by Evans and Archer (1968) and Elton and Gruber (1977) to a systemic context. This allows to better understand the controversial relation between the firm capital structure and diversification (Jensen and Meckling, 1976). Finally, our work complements existing theoretical literature that, after the 2007-2008 financial crisis, started to investigate the role of risk diversification for the stability of the financial system (Battiston et al., 2009a; Brock et al., 2009; Ibragimov and Walden, 2007; Stiglitz, 2010, Wagner, 2009). Our results about a critical level of diversification can be used for improving macro-prudential regulations, for example by enforcing a ceiling to excessive leverage.

\section{Model}

\subsection{Banks and their portfolio}

We extend the Merton (1974) framework to a set of $M$ banks such that for each bank $i \in M$ the following balance-sheet identity holds true at any time $t$

$$
a_{i}(t)=h_{i}(T)+e_{i}(t)
$$

where $a_{i}(t)$ is the market value of bank $i$ 's assets. $h_{i}(T)$ is the promised payment of its liabilities at maturity date $T$. 1 Finally, $e_{i}(t)$ is the equity value which keeps the two sides of the balance sheet even. The debt-to-asset ratio, $h_{i}(T) / a_{i}(0):=f_{i}(0)=f_{i} \in(0,1)$ captures the different capital structure between banks and $1 / f_{i}$ can be seen as an approximate measure of the credit quality of a bank.

The objective of a risk-averse bank is to increase its asset value per unit of risk. The latter results from investing in a number non-divisible risky projects $l \in\{1,2,3, \ldots, N\}$, i.e. activities related to the real-economy such as loans to firms and households, infrastructure projects, or real-estate investments. Each of these projects has a certain price value per unit, $\nu_{l}(t)$ at time $t$. If this price has decreased compared to the initial price $\nu_{l}(0)$, banks face a loss, and they gain in the opposite case.

\footnotetext{
${ }^{1}$ The equation indirectly assumes only one seniority of bonds with the same maturity date $T$.
} 
If at time $t=0$ bank $i$ acquires $x_{i l}(0)$ units of project $l$ at a price $\nu_{l}(0)$, its asset is given by:

$$
a_{i}(0):=\sum_{l=1}^{n_{i}} x_{i l}(0) v_{l}(0)
$$

The investment position of bank $i$ at $t=0$ is denoted by the vector $\mathbf{x}_{i}(0):=\left\{x_{i 1}(0), \ldots, x_{i l}(0)\right\}$ of risky projects. In this paper, we assume that bank $i$ invests the same proportion of its assets in each of the projects, i.e. $a_{i}(t) / n_{i}=$ const. for all $t \geq 0$, which implies that Eq. (2) simplifies to $a_{i}(0)=n_{i} x_{i l}(0) \nu_{l}(0)$, which holds for every project $l$ bank $i$ invested in. Consequently, each bank initially has a different leverage ratio $f_{i}$ and a different diversification expressed by the number $n_{i}$ of projects to invest:

$$
\frac{1}{f_{i}}=\frac{n_{i} x_{i l}(0) v_{l}(0)}{h_{i}(T)}
$$

We have assumed here that diversification is not with respect to the type of projects chosen, but only with respect to their number. That means that projects, from this perspective, are indistinguishable and uncorrelated, so we also assume that they have the same initial price $\nu_{l}(0)=\nu_{0}$.

The balance-sheet identity (1) has to be guaranteed at any time $t$, which implies $a_{i}(t)=$ $n_{i} x_{i l}(t) \nu_{l}(t)$ for the asset side to hold true. Since the fraction of assets allocated to each project is kept constant over time, bank $i$ has to adjust the number of units $x_{i l}(t)$ in its portfolio with respect to the price changes $\nu_{l}(t)$. In practice, at each trading date, the asset allocation is revised such that, after rebalancing, the amount invested in each of the projects is again $a_{i}(t) / n_{i} \mathrm{~L}^{2}$ That is, bank $i$ holds the investment position $\mathbf{x}_{i}(t)$ during the period $[t-d t, t]$ and liquidates it at the prevailing prices at time $t$. Simultaneously bank $i$ sets up the new portfolio $\mathbf{x}_{i}(t+d t)$. Therefore, $\mathbf{x}_{i}$ represents a "contrarian" asset allocation strategy because the portfolio is rebalanced by selling "winners" and buying "losers" at any time $t$ according to the formula

$$
x_{i l}(t+d t)=\frac{1}{\nu_{l}(t+d t)} \frac{1}{n_{i}} \sum_{k=1}^{n_{i}} x_{i k}(t) \nu_{k}(t+d t)
$$

for each project $l$ in $\mathbf{x}_{i}(t)$. As in Merton (1974), the price dynamics is given by a Geometric Brownian Motion of the form

$$
\frac{d \nu_{l}}{\nu_{l}(t)}=\mu d t+\sigma d \tilde{B}_{l}(t), \quad l=1, \ldots, N
$$

The random shocks $\tilde{B}_{l}(t)$ follow a standard Brownian motion defined on a complete filtered probability space $\left(\Omega ; \mathcal{F} ;\left\{\mathcal{F}_{t}\right\} ; \mathbb{P}\right)$, with $\mathcal{F}_{t}=\sigma\{\tilde{B}(s): s \leq t\}$ and $\mathbb{E}\left(d \tilde{B}_{l}, d \tilde{B}_{y}\right)=0$ for all pairs $l, y$ in the set of risky projects. The drift term $\mu$ describes the instantaneous risk-adjusted expected

\footnotetext{
${ }^{2}$ The theory of rebalanced portfolios is developed in Kelly Jr (1956) and Mossin (1968), among the others.
} 
growth rate, and $\sigma>0$ is the volatility of the growth rate. Both $\mu$ and $\sigma$ are the same for all projects because they are indistinguishable. Despite projects are uncorrelated, diversification increases the likelihood of having overlapping portfolios between bank $i$ and $j$, i.e. $\mathbb{E}\left(d B_{i}, d B_{j}\right)=$ $\rho_{i j} d t$, where $\rho_{i j}$ is the asset correlation in the portfolios of banks $i$ and $j$.

Using the expression $a_{i}(t)=n_{i} x_{i l}(t) \nu_{l}(t)$, we arrive after some transformations at the following dynamics for the asset side of bank $i$ :

$$
\frac{d a_{i}}{a_{i}(t)}=\mu d t+\frac{\sigma}{\sqrt{n_{i}}} d B_{i}(t), \quad \text { where } d B_{i} \sim N(0, d t) .
$$

For each bank $i$ the dynamics is then as follows: initially it selects a certain strategy, defined as $\left(f_{i}, n_{i}\right)$, which contains the chosen leverage $f_{i}$ and the number $n_{i} \leq N$ of risky projects, in each of which it invests the same proportion of assets. The bank holds the selected portfolio and locks the proportion of assets allocated to each project until time $T$. Given the stochastic nature of the price dynamics and the risk associated with this, the bank has to estimate the optimal combination of leveraging and diversification across the set of $N$ risky projects. This problem will be addressed in the next section.

\subsection{Individual and Systemic Default Probability}

Following the structural approach proposed by Merton (1974), we assume that the default occurs if the asset value of bank $i$ at debt maturity date $T$ falls beneath the book value of its debt, i.e., $a_{i}(T) \leq h_{i}(T)$. More formally, given the probability space $(\Omega, \mathcal{F}, \mathbb{P})$, the default probability $\mathbb{P} D_{i}\left(f_{i}, n_{i}\right)$ of the bank $i$ using strategy $\left(f_{i}, n_{i}\right)$ is at the end of the period $T$ defined as:

$$
\mathbb{P} D_{i}\left(f_{i}, n_{i}\right):=\mathbb{P}\left[a_{i}(t) \leq h_{i} \mid a_{i}(0)=a_{i 0}\right]=\mathbb{P}\left[\ln \left(a_{i}(t)\right) \leq \ln \left(h_{i}\right) \mid a_{i}(0)=a_{i 0}\right]=\Phi_{1}\left(z_{i}\right)
$$

where $\Phi_{1}\left(z_{i}\right)$ is the standard cumulative normal distribution function with the argument

$$
z_{i}=-\frac{\ln \left(1 / f_{i}\right)+\mu T-\chi / n_{i}}{\sqrt{2 \chi / n_{i}}} ; \quad \chi=\frac{\sigma^{2} T}{2}
$$

The constant $\chi$ combines the effects of asset volatility and time to maturity. The probability of bankruptcy depends on the distance between the current asset value $a_{i}(t)$ and the debt value $h_{i}(T)$, adjusted for the expected growth in asset value, $\mu$, relative to asset volatility, $\sigma$. We verify that the default probability is increasing in $h_{i}$, decreasing in $a_{i}$ and, for $a_{i}>h_{i}$, increasing in $\sigma / \sqrt{n_{i}}$, which is in line with economic intuition. Then, increasing asset diversification (i.e., letting $\left.n_{i} \rightarrow N\right)$ is desirable since it lowers $\Phi_{1}\left(z_{i}\right)$, ceteris paribus.

The individual default probability (7) refers to the default of a bank irrespective of the default of other banks $3^{3}$ However, in order to quantify systemic risk, we have to specifically address the

\footnotetext{
${ }^{3}$ Indirect effects such as price movements caused by the default of other banks are not considered here.
} 
impact of the default of one bank on another bank. In this paper, we extend the approach by Merton (1974) by calculating this impact based on the above considerations. For simplicity, we consider only two banks in the first place and further assume the expected growth to be zero, $\mu=0$. A possible extension of the framework to $M$ banks is feasible (see e.g., Cathcart and El-Jahel (2004)) and is further discussed in Section 3. Also the systemic effects of a "booming" market, $\mu>0$, compared to those of a "crashing" market, $\mu<0$, are discussed later.

Systemic default occurs whenever banks 1 and 2 jointly default at the end of period $T$. More formally, given the probability space $(\Omega, \mathcal{F}, \mathbb{P})$, the systemic default probability $\mathbb{P} S\left(n_{1}, f_{1} ; n_{2}, f_{2}\right)$ of the banking system consisting of two banks $i \in\{1,2\}$ is defined as:

$$
\mathbb{P} S\left(n_{1}, f_{1} ; n_{2}, f_{2}\right):=\Phi_{2}\left(z_{1}, z_{2}, \rho_{1,2}\right)
$$

$\Phi_{2}$ is the standard bivariate cumulative normal distribution function and $\rho_{1,2}$ is the asset correlation between bank 1 and 2, which results from the fact that both may have invested into the same risky project.

The calculation of Eq. 9 implies that we have to integrate the underlying density function $g\left(z_{1}, z_{2}, \rho_{1,2}\right)$ over a two-dimensional grid $\left(z_{1}, z_{2}\right)$, to obtain the cumulative function $\Phi_{2}$. This is generally not simple, especially when the asset correlation $\rho_{1,2}$ is non-constant. Various tables exist if $g$ is a bivariate normal distribution (Owen, 1962, Pearson, 1931). Because this does not apply to our case, we use a method proposed by Jantaravareerat (1998), which allows us to calculate $\Phi_{2}$ as follows:

1. Calculate the joint probability density function $g\left(z_{1}, z_{2}, \rho_{1,2}\right)$ defined as:

$$
g\left(z_{1}, z_{2}, \rho_{1,2}\right)=\frac{1}{2 \pi \sqrt{1-\rho_{1,2}^{2}}} \exp \left\{-\frac{1}{2\left(1-\rho_{1,2}^{2}\right)}\right\}\left(z_{1}^{2}-2 \rho_{1,2} z_{1} z_{2}+z_{2}^{2}\right)
$$

where the $z_{i}$ are given in Eq. (8). This returns the joint probability density for a square grid with a side length, $N$, spanned by the range of $z_{1}$ and $z_{2}$.

2. Map the sorted values of $z_{1}, z_{2}$ to the index sets $I_{z_{1}}$ and $I_{z_{2}}$. I.e, the elements of $I_{z_{1}}$ and $I_{z_{2}}$ are $y_{1}$ and $y_{2}$ that range from $[1,1]$ to $[N, N]$. Hence, after calculating the density $g\left(z_{1}, z_{2}, \rho_{1,2}\right)$, we map it to $g\left[y_{1}, y_{2}\right]^{4}$ by using the row and column coordinates $\left[y_{1}, y_{2}\right]$.

3. Calculate the mean density $d\left[y_{1}, y_{2}\right]$ at each cell as the mean of itself and its three immediate neighbors.

$$
d\left[y_{1}, y_{2}\right]=\frac{1}{4}\left(g\left[y_{1}, y_{2}\right]+g\left[y_{1}+1, y_{2}\right]+g\left[y_{1}, y_{2}+1\right]+g\left[y_{1}+1, y_{2}+1\right]\right)
$$

\footnotetext{
${ }^{4}$ Square brackets shall denote that we use the index instead of the actual values as coordinates
} 
4. Calculate the volume of each cell, $P\left[y_{1}, y_{2}\right]$ by multiplying the average density by the area, to accommodate for the fact that the indexed cells were originally of unequal size:

$$
P\left[y_{1}, y_{2}\right]=d\left[y_{1}, y_{2}\right]\left(z_{2}-z_{1}\right)^{2}
$$

5. Calculate the joint distribution function, $\Phi_{2}\left[y_{1}, y_{2}\right]$ for each cell $\left[y_{1}, y_{2}\right]$ by summing up the volumes of all cells up to this point,

$$
\Phi_{2}\left[y_{1}, y_{2}\right]=\sum_{j_{1}=1}^{y_{1}} \sum_{j_{2}=1}^{y_{2}} P\left[j_{1}, j_{2}\right]
$$

6. Map $\Phi_{2}\left[y_{1}, y_{2}\right]$ back to $\Phi_{2}\left(z_{1}, z_{2}, \rho_{1,2}\right)$ by using the index sets $I_{z_{1}}, I_{z_{2}}$

In the following section we analyze Eq. (13), to quantify the impact of the interplay between diversification $\left(n_{1}, n_{2}\right)$ and leverage $\left(f_{1}, f_{2}\right)$ on the systemic default probability. Instead of the general case, where both banks follow different strategies $\left(f_{i}, n_{i}\right)$, we restrict our calculation to the homogeneous case, i.e. we assume the same leverage and diversification for both banks, i.e. $f_{1}=f_{2}=f$ and $n_{1}=n_{2}=n$. In Section 3, we will also comment on the more general case, to clarify that the homogeneous assumption is not a severe restriction. For the homogeneous case, the asset correlation becomes a constant expressed as

$$
\rho_{1,2}=\frac{n^{2}}{N} \frac{\sigma^{2}}{n^{2}} / \frac{\sigma^{2}}{n}=\frac{n}{N}
$$

This reduces the problem to the discussion of $\Phi_{2}(z, \rho)$ with $z(f, n, \chi)$ given by Eq. (8).

\section{Analysis}

The emphasis of this section is to quantify the impact of banks' strategy $(f, n)$, in the homogeneous case, on the systemic default probability $\Phi_{2}(z(f, n, \chi), n / N)$, given some properties of the market, $\chi$. In detail, we assess the benefits of diversification to eliminate the portion of systemic risk that is caused by excessive leverage. The latter is defined as the difference $\Delta f:=f_{a}-f_{n}$, where $f_{n}$ refers to the "normal" market level of leverage and $f_{a}>f_{n}$ to the "abnormal" level. We then calculate the difference $\Delta \Phi_{2}\left(f_{a}, f_{n}\right):=\Phi_{2}\left(f_{a}\right)-\Phi_{2}\left(f_{n}\right)$ (all other parameters fixed). $\Delta \Phi_{2}$ gives us the increase in systemic risk associated with excessive leverage, i.e., it can be only positive (increasing risk) or zero (no impact of the leverage), but not negative because increasing leverage should not result in decreasing risk. In Figure 1 we plot $\Delta \Phi_{2}$ over a parameter sweep of the market size, $N$, the market risk, $\chi$, and the degree of diversification, $n$. Figure 1 demonstrates the impact of the parameters $N, n$, and $\chi$ on the systemic risk for two different sets of leverage 
values: $\left\{f_{n}, f_{a}\right\}=\{0.10,0.25\}$ with $\Delta f=0.15$ and $\left\{f_{n}, f_{a}\right\}=\{0.25,0.50\}$ with $\Delta f=0.25$. In the graph, we only distinguish between zerd 5 (gray) and positive (black) values of $\Delta \Phi_{2}$. From this, we can draw the following conclusions.
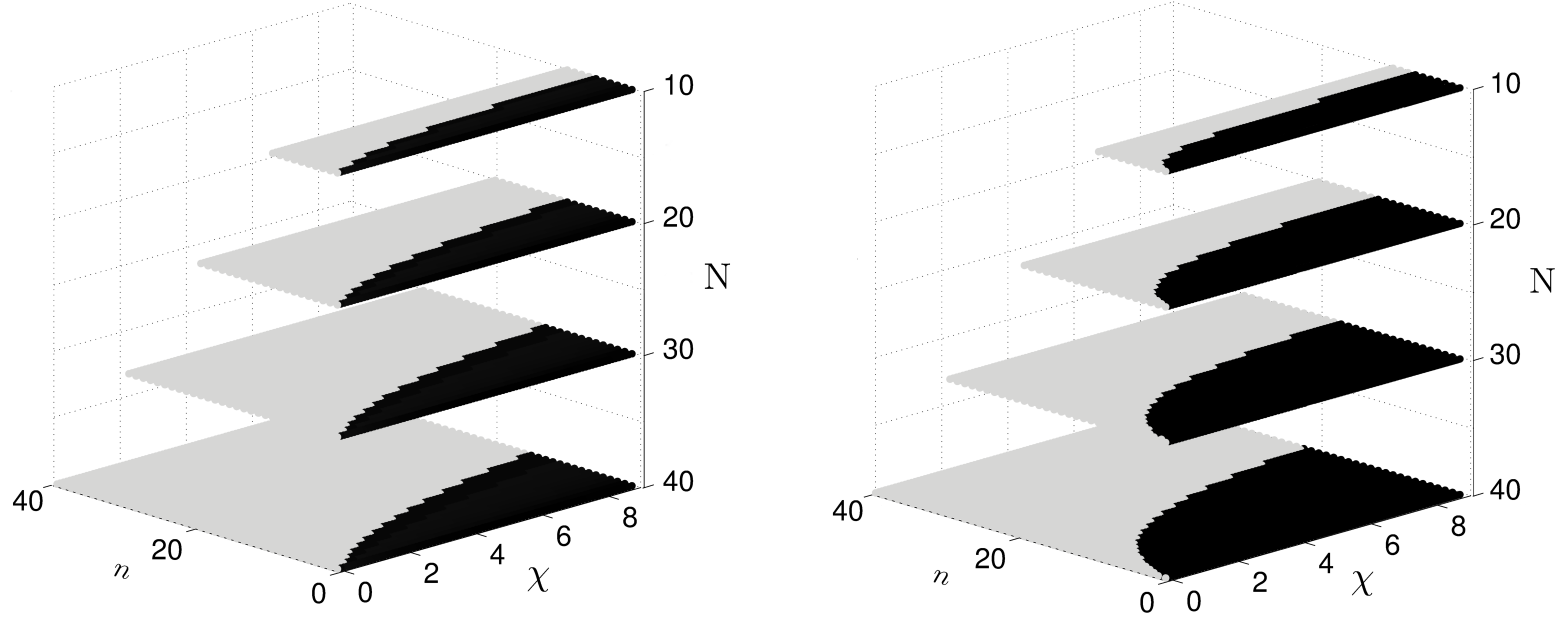

Figure 1: Effects of market size, degree of diversification, and leverage on the systemic default probability. The two plots show the difference $\Delta \Phi_{2}\left(f_{a}, f_{n}\right)$. Left: $\left\{f_{n}, f_{a}\right\}=\{0.10,0.25\}$ and $\Delta f=0.15$. Right: $\left\{f_{n}, f_{a}\right\}=\{0.25,0.50\}$ and $\Delta f=0.25$. Each plane corresponds to a particular market size, $N$. Black color indicates positive values of $\Delta \Phi_{2}$, whereas gray color indicates zero. Other parameters: $N \in\{10,20,30,40\}, n \in[1, N], \chi \in[0.001,9]$.

Diversification: For all other values constant, there exists a diversification level $n^{\star}\left(f_{n}, f_{a}, N, \chi\right)$ beyond which excessive leverage does not result in an increasing systemic default probability $\Delta \Phi_{2}$. Remarkably, $n^{\star}<<N$ (see the lines that separate the black from the gray areas in Fig. 11. In other terms, conditional on the market size $N$ and market properties $\chi, n^{\star}$ represents the minimum number of risky projects each bank has to hold in order to prevent the increase of systemic risk from excessive leverage. As Figure 1 indicates, $n^{\star}$ increases monotonously with market size $N$, i.e., a bigger market requires more diversification. Moreover, we can show that $n^{\star}$ increases for "crashing" markets, $\mu<0$, and decreases for "booming" markets, $\mu>0$. This is in line with economic intuition, i.e. diversification works well only in a booming market, (Tasca and Battiston, 2011). The specific dependence $n^{\star}\left(f_{n}, f_{a}, N, \chi\right)$ can be obtained only numerically. Table 1 gives some numbers for the minimum value, in accordance with Figure 1.

\footnotetext{
${ }^{5}$ We talk about small positive values of $-10^{-6}$ or smaller, which are approximately zero, compared to an order of -10 for the black area.
} 


\begin{tabular}{c|ccc|ccc}
\hline \hline & $\multicolumn{3}{|c|}{f_{a}, f_{b}}=\{0.1,0.25\}$ & $\multicolumn{3}{c}{f_{a}, f_{b}}=\{0.25,0.5\}$ \\
$N$ & $n^{\star}(1.6)$ & $n^{\star}(5.1)$ & $n^{\star}(8.9)$ & $n^{\star}(1.6)$ & $n^{\star}(5.1)$ & $n^{\star}(8.9)$ \\
\hline 10 & 3 & 5 & 6 & 5 & 6 & 7 \\
20 & 4 & 8 & 10 & 8 & 11 & 12 \\
30 & 5 & 10 & 13 & 10 & 15 & 17 \\
40 & 5 & 11 & 15 & 12 & 18 & 22 \\
\hline \hline
\end{tabular}

Table 1: Minimum level of diversification $n^{\star}(\chi)$ to eliminate the increase of systemic risk due to excessive leverage. Left: $\left\{f_{a}, f_{b}\right\}=\{0.1,0.25\}$ and $\Delta f=0.15$. Right: $\left\{f_{a}, f_{b}\right\}=\{0.25,0.5\}$ and $\Delta f=0.25$. Other parameters: $N \in\{10,20,30,40\}, \chi \in\{1.6,5.1,8.9\}$. The numbers for $n$ are rounded to the nearest integers.

Market size: We can observe both from Figure 1 and from Table 1 that an increasing market size $N$ results in an increasing number of projects to be chosen and hold for optimal diversification. Hence, larger markets bear more risk which has to be compensated. Indeed, despite the fact that broader markets in which banks can choose from a bigger pool of projects allow for better diversification possibilities, they also reduce the effectiveness of diversification. That is because the boundary $n^{\star}$ increases with the market size. In this context, systemic risk can be hardly contained through diversification. It could be more interesting, however, to discuss the relative risk, i.e. the ratio of the black area (where systemic risk has increased with leverage increase) over the gray area (where systemic risk is independent of leverage and diversification). It appears that the relative risk decreases with increasing market size. I.e., for larger markets it becomes more likely to invest in projects such that banks 1 and 2 become independent enough with respect to their diversification strategy. For $n$ randomly chosen projects, a larger market reduces the risk of systemic default, provided that $n$ is above the critical number $n^{\star}$.

Leverage and market risk: Comparing both sides of Figure1, we observe that the black area increases if the excessive leverage $\Delta f$ increases. This also holds for increasing absolute leverage values (not shown). However, it is worth to note that the impact of leverage is not as severe as one might expect. Its influence becomes noticeable mainly for higher values of $\chi$, i.e. for extreme market risks. Instead, in less volatile markets, i.e. for moderate market risks, the relative impact of the leverage decreases.

The main message drawn from the above consideration is the emergence of two market regimes: the safe regime in which excessive leverage in the market does not increase systemic risk (gray areas) and the risky regime in which excessive leverage in the market does increase systemic risk (black areas). There are two complementary ways to move from the risky to the safe regime. 
First, for a given market condition $\chi$, banks should increase their level of diversification at least up to $n^{\star}$ (outward movement along the $n$ axis in Fig. 1). Second, for a given diversification level $n$, the market should become less risky (inward movement along the $\chi$ axis in Fig. 1).

Our results already allow us to discuss changes in the basic assumptions that underlie the Merton (1974) model. So far, we have assumed that there are no correlations between projects. If these are taken into account, the boundary $n^{\star}$ should rise dramatically to a level that may be difficult to achieve by some market participants, especially if the market size is very big. This effect should even further increase if we take into account the costs for monitoring and transaction of the projects. Such costs are expected to increase with $n$ and thus also lead to an increase in $n^{\star}$. Therefore, the estimation given in this paper should be considered as the minimum value. At the same time, this is also the optimal diversification level because it warrants to be in the safe regime at the minimal cost.

Our model could be extended to the more general case of $M$ banks, which may be heterogeneous in terms of leverage and diversification strategies $\left(f_{i}, n_{i}\right)$. This would lead to an increasing complexity of the analysis. However the general findings of the paper would qualitatively remain the same because such generalizations do not increase the degree of freedom for the banks, i.e. their set of strategies. At this point, we can already argue that the impact of the heterogeneity in strategies is as follows: If some banks choose a high level of leverage, this increases $n^{\star}$. This in turn forces those other banks with lower leverage to choose a larger diversification level, to compensate for this, i.e. it creates negative externalities in the system.

\section{Conclusions}

Financial institutions such as banks use different strategies to run their businesses. In this paper, we consider two of them, namely leverage and diversification. The first one allows banks to increase their expected return on equity based on debt financed investments. The second one is used to mitigate losses from such investments. Hence, both strategies have a different impact on the default probability of a bank: leverage amplifies the default risk, while diversification reduces it. In a finite market banks cannot choose completely independent diversification strategies. Instead, their portfolios overlap with diversification, which correlates the default probability of individual banks.

In this paper, we are interested in the impact of these strategies on the systemic default probability, i.e. the probability of joint defaults. In particular, we answer the question if and how an increase of individual risk, due to excessive leverage, leads to an increase of the systemic default probability, and if and how this can be mitigated by an optimal diversification level. As the main result, we determine the boundary between two market regimes: a safe regime in which excess 
leverage can be mitigated by better diversification, thus not resulting in an increase of systemic risk, and a risky regime in which this is not possible resulting in increased systemic risk.

Our findings contribute to the ongoing discussions of the optimal regulatory framework for financial markets. The question, whether benefits from diversification can offset the systemic risk, due to excessive leverage, can be answered by means of our distinction between risky and safe conditions. This also extends to the question whether capital requirements should be lowered for institutions with more diversified investments. Here, we show that a critical level of diversification exists which depends on the market size and the market conditions and thus needs to be monitored to prevent a transitions toward the risky regime. Such insights can be used to improve macro-prudential regulations, such as the current Basel III risk-based framework, which is portfolio invariant, i.e., does not consider the role for diversification. As a conclusion from our results, portfolio invariance leads to a significantly higher maximum leverage ratio allowed for banks in the current regulatory framework, i.e. it can result in a much riskier financial system.

\section{References}

Battiston, S., Gatti, D. D., Gallegati, M., Greenwald, B. C. N., and Stiglitz, J. E. (2009a). Liaisons dangereuses: Increasing connectivity, risk sharing and systemic risk. NBER Working Paper Series n.15611.

Battiston, S., Lorenz, J., and Schweitzer, F. (2009b). Failure cascades on networks. Submitted.

Board, F. S. (2010). Guidance to Assess the Systemic Importance of Financial Institutions, Markets and Instruments: Initial Considerations: Report to the G-20 Finance Ministers and Central Bank Governors. Staff of the International Monetary Fund and the Bank for International Settlements, and the Secretariat of the Financial Stability Board.

Bohn, J. R. (2000). A survey of contingent-claims approaches to risky debt valuation. The Journal of Risk Finance, 1(3):53-70.

Brock, W., Hommes, C., and Wagener, F. (2009). More hedging instruments may destabilize markets. Journal of Economic Dynamics and Control, 33(11):1912-1928.

Cathcart, L. and El-Jahel, L. (2004). Multiple defaults and merton's model. The Journal of Fixed Income, 14(1):60-68.

Elton, E. J. and Gruber, M. J. (1977). Risk reduction and portfolio size: An analytical solution. The Journal of Business, 50(4):pp. 415-437.

Embrechts, P., Klüppelberg, C., and Mikosch, T. (2011). Modelling extremal events: for insurance and finance, volume 33. Springer. 
Evans, J. and Archer, S. (1968). Diversification and the reduction of dispersion: an empirical analysis. The Journal of Finance, 23(5):761-767.

FSA (2009). The turner review: a regulatory response to the global banking crisis. financial service authority. Financial Service Authority.

FSB (2009). Report of the financial stability forum on addressing procyclicality in the financial system. Financial Stability Forum.

Ibragimov, R. and Walden, J. (2007). The limits of diversification when losses may be large. Journal of Banking and Finance, 31(8):2551-2569.

Jantaravareerat, M. (1998). Approximation of the Distribution Function for the Standard Bivariate Normal. Ph.D Thesis. Illinois Institute of Technology, Chicago, IL.

Jensen, M. C. and Meckling, W. H. (1976). Theory of the firm: Managerial behavior, agency costs and ownership structure. Journal of Financial Economics, 3(4):305 - 360.

Kelly Jr, J. (1956). A new interpretation of information rate. Information Theory, IRE Transactions on, 2(3):185-189.

Kiyotaki, N. and Moore, J. (2002). Balance-Sheet Contagion. American Economic Review, 92(2):46-50.

Merton, R. (1974). On the Pricing of Corporate Debt: The Risk Structure of Interest Rates. The Journal of Finance, 29(2):449-470.

Mossin, J. (1968). Optimal multiperiod portfolio policies. The Journal of Business, 41(2):215229.

Owen, D. (1962). Handbook of Statistical Tables. Addison-Wesley Pubblishing Company, Reasing, Mass.

Pearson, K. (1931). Table for Statiticians and Biometricians, Part II. Cambridge University Press.

Stein, J. (2009). Presidential address: Sophisticated investors and market efficiency. The Journal of Finance, 64(4):1517-1548.

Stiglitz, J. E. (2010). Risk and global economic architecture: Why full financial integration may be undesirable. NBER Working Paper No. 15718.

Tasca, P. and Battiston, S. (2011). Diversification and financial stability. CCSS Working Paper.

Wagner, W. (2009). Diversification at financial institutions and systemic crises. Journal of Financial Intermediation. 